\title{
Atrial Fibrillation Ablation Success Defined by Duration of Recurrence on Cardiac Implantable Electronic Devices
}

\author{
Graham Lohrmann ${ }^{1}$, Rachel Kaplan ${ }^{2}$, Paul Ziegler ${ }^{3}$, João Monteiro ${ }^{4}$, and Rod Passman ${ }^{1}$ \\ ${ }^{1}$ Northwestern University Feinberg School of Medicine \\ ${ }^{2}$ Northwestern University, Feinberg School of Medicine \\ ${ }^{3}$ Medtronic, Inc. \\ ${ }^{4}$ Medtronic Inc
}

August 23, 2020

\begin{abstract}
Background: Ablation for atrial fibrillation (AF) has emerged as an effective method of rhythm control. This exploratory analysis aimed to determine how various measures of recurrence would influence the definition of treatment success. Methods: Using an EHR dataset from 01/2007-06/2019 linked with Medtronic cardiac implantable electronic device (CIED) data, patients who underwent a first AF ablation procedure following CIED implantation were identified. Data were analyzed for recurrence of AF stratified by varying definitions of successful ablation. Performance of various simulated external AF monitoring strategies was assessed. Results: A total of 665 patients were analyzed including 248 with paroxysmal AF (mean age $66.2 \pm 9.3$ years, $73.0 \%$ male) and 417 patients with persistent AF (mean age $67.3 \pm 9.0$ years, $73.6 \%$ male). Patients with paroxysmal AF, survival free from recurrence at 1 year ranged from $28.2 \%$ to $72.1 \%$ ( $>6 \mathrm{~min}$ and $>23$ hours thresholds, respectively) with an overall median percentage of time in AF reduction of $99.6 \%$. Patients with persistent AF, survival free from recurrence at 1 year ranged from $24.9 \%$ to $60.0 \%$ ( $>6$ min and 7 consecutive days $>23$ hours thresholds, respectively) with an overall median percentage of time in AF reduction of 99.3\%. A single 7-day monitoring strategy had a sensitivity of $<50 \%$ for detecting AF $>6$ min in patients with paroxysmal and persistent AF. Conclusion: In this real-world dataset of AF patients with CIEDs undergoing catheter ablation, treatment success varied substantially with different definitions of minimally required AF duration and is significantly impacted by the method of recurrence detection.
\end{abstract}

\section{Introduction}

Catheter ablation for the treatment of atrial fibrillation (AF) has emerged as a safe ${ }^{1}$ and superior strategy compared to antiarrhythmic drug therapy in reducing AF recurrence ${ }^{2,3,4}$, improving quality of life ${ }^{5,6}$, and reducing mortality and hospitalizations in patients with heart failure and reduced ejection fraction ${ }^{7,8}$. Expert consensus statements recommend that recurrence of AF be defined as any AF, atrial tachycardia (AT) or atrial flutter (AFL) of at least 30 seconds duration detected more than 3 months (i.e. the blanking period) following catheter ablation ${ }^{9}$. Success of the procedure varies depending on how success is defined, with significant differences in outcome definitions based on required AF duration as well as the monitoring strategy employed to detect treatment failure ${ }^{10,11,12,13}$. Furthermore, some authors have suggested that a reduction in AF burden may be a more objective endpoint rather than a binary outcome of recurrence as a measure of success $^{14}$. This may be even more important given emerging data correlating AF duration and burden with risk of stroke ${ }^{15,16,17,18}$, a risk which may be attenuated in part by AF ablation ${ }^{19}$. The present exploratory analysis used a large de-identified electronic health record (EHR) dataset to assess how variations in defining $\mathrm{AF}$ recurrence and frequency and duration of monitoring influence an assessment of treatment success or failure. 


\section{Methods}

Study Population: Using patient-level data from the Optum $囚$ de-identified EHR dataset from 01-012007-to 06-30-2019 linked with Medtronic cardiac implantable electronic device (CIED) data, patients who underwent an AF ablation procedure following CIED implantation were identified. Only the first AF ablation procedure observed in the dataset was considered. Following a 90-day blanking period from the time of the first catheter AF ablation procedure observed in the dataset, patient level daily AT/AF burden data (collected by the CIED) was analyzed for AF recurrence using varying definitions of recurrence, which were then compared against simulated external monitoring strategies used clinically to detect recurrence.

Inclusion and Exclusion criteria: Patients were required to have an AF ablation procedure (identified by the CPT codes $93656 \&$ 93657), and only the patient's first AF ablation procedure observed in Optum $\mathbb{R}$ dataset was considered. Patients were also required to have a Medtronic CIED capable of continuous AF monitoring implanted prior to the AF ablation procedure, have at least 6 months between first record in the Optum EHR and the Medtronic CIED implant date, have daily AT/AF burden available for every day during a period of 30 days prior to $\mathrm{AF}$ ablation procedure, with at least one day having greater than 23 hours of available burden data, and have at least 1 daily AT/AF burden measurement available after the end of the 90-day blanking period.

Medical history prior to AF ablation: History of hypertension, prior stroke/TIA, heart failure, diabetes, and vascular diseases were extracted from the Optum $\mathbb{R}$. EHR data based on the presence of diagnosis codes (ICD-9/ICD-10) in the diagnosis table on or prior to the AF ablation procedure date. The list of codes considered for each of these conditions is available in Supplement Table 1.

AF type prior to AF ablation: Patients were classified as having persistent AF if they had 7 consecutive daily AT/AF burden $>23$ hours in the 30 days prior to the AF ablation procedure; all other patients were considered to have paroxysmal AF.

Atrial Fibrillation Recurrence: AF recurrence definitions included daily AT/AF burden duration of at least 6 min, 1 hour, 6 hours, 12 hours, 23 hours, 7 consecutive days with $>23$ hours, and a rolling 30day average burden $>3.6$ hours ( $0.5 \%$ burden). Kaplan-Meier estimator was used to estimate the survival probability for each recurrence definition post-ablation following a 90-day blanking period.

Atrial Fibrillation Burden Reduction Following Ablation: Two metrics were considered to quantify the change in AF burden following ablation: percent of time in $\mathrm{AT} / \mathrm{AF}$ and median daily $\mathrm{AT} / \mathrm{AF}$ duration.

For each patient, the percent of time in AT/AF was computed as the division of the total AT/AF burden hours by the total observed time, while the median daily AT/AF was the median duration of the observed daily AT/AF burden measurements. Both metrics were computed stratifying by the time period with respect to ablation procedure: pre-ablation (all observed daily AT/AF burden measurements 1 year prior to the AF ablation procedure), and post ablation (all daily AT/AF burden measurements observed between the days 90 and 455 (i.e. one-year post-blanking period) post AF ablation).

Monitoring Strategy Simulation: Using daily AT/AF burden measurements as the source of truth,

sensitivity and negative predictive value (NPV) were computed to assess performance of various external AF monitoring strategies used in clinical practice with respect to the detection of events of daily AT/AF burden $>6$ min, daily AT/AF burden $>6$ hours and daily AT/AF burden $>23$ hours. The simulated monitoring strategies were: 24 -hour, 48-hour, 7-day, 21-day, 30-day, quarterly 24-hour, quarterly 48 -hour, quarterly 7 day and monthly 24 -hour continuous external monitoring. All these monitoring strategies were considered to occur within 1 year after the 90-day blanking period and 100\% patient compliance with external monitoring was assumed. Only data from patients who had uninterrupted daily AT/AF burden measurements during this time period were included in the simulation. Lastly, a bootstrap method (with 10,000 samples) was used to build $95 \%$ confidence intervals (CI) for the performance metrics. The initial day of the AF monitoring strategy was randomly assigned within the first two weeks post blanking period for each patient. 


\section{Results}

Baseline characteristics: There were 57,206 patients who had at least one record indicating an AF ablation procedure, 2,402 (4.2\%) of which had a Medtronic CIED implanted prior to the first AF ablation procedure on record, and $665(1.2 \%)$ of which satisfied the full inclusion/exclusion criteria. Of those, $417(62.7 \%)$ patients had persistent $\mathrm{AF}$ (mean age $67.3 \pm 9.0,73.6 \%$ male) and $248(37.3 \%$ ) patients had paroxysmal AF (mean age $66.2 \pm 9.3,73.0 \%$ male) as defined by their AF pattern within 30 days prior to ablation (Table 1). The mean time between AF ablation procedure and last observed daily AF burden was $22.7 \pm 14.7$ months.

Recurrence of Atrial Fibrillation: In patients with paroxysmal AF (Figure 1, Panel A), survival free from $\mathrm{AF}$ recurrence at 1-year post blanking period varied from $28.2 \%$ (using a definition of AF recurrence of $>6$ minutes) to $72.1 \%$ (using the definition $>23$ hours). Similarly, in patients with persistent AF, survival free from $\mathrm{AF}$ recurrence at 1-year post blanking period ranged from $24.9 \%$ (using a definition of $\mathrm{AF}$ recurrence of $>6$ minutes) to $60.0 \%$ (using the definition of 7 consecutive days $>23$ hours; Figure 1, Panel B).

Atrial Fibrillation Burden Reduction Following Ablation: Of the 248 patients with paroxysmal AF, the, overall median percentage of time in $\mathrm{AF}$ was reduced by $99.6 \%$ (from $23.7 \%$ prior to ablation to $0.1 \%$ following ablation) and the overall median daily AF burden was reduced from 0.28 to 0 hours (Figure 2). Of the 417 patients with persistent $\mathrm{AF}$ at baseline, the overall median percentage of time in $\mathrm{AF}$ was reduced by $99.3 \%$ (from $67.5 \%$ prior to ablation to $0.5 \%$ following ablation) and the overall median of median daily AF burden was reduced from 23.98 to 0 hours (Figure 3).

Monitoring Strategy Simulation: Out of the 665 patients who satisfied the inclusion/exclusion criteria, $369(55.5 \%)$ had at least 1 year of uninterrupted daily AF observations (thus included in the monitoring strategy simulation), of which 137 had paroxysmal AF and 232 had persistent AF at baseline. Of the 138 patients with paroxysmal AF at baseline, 101 (73.7\%), $72(52.6 \%)$ and $41(29.9 \%)$ had a least one day with $\mathrm{AF}>6$ min, $\mathrm{AF}>6$ hours and $\mathrm{AF}>23$ hours, respectively within 1-year post blanking period. Of the 232 patients with persistent AF at baseline, 174 (75.0\%), 134 (57.8\%) and 112 (48.3\%) had a least one day with $\mathrm{AF}>6 \mathrm{~min}, \mathrm{AF}>6$ hours and $\mathrm{AF}>23$ hours respectively within 1-year post blanking period. Sensitivity and NPV estimates for all monitoring strategies are graphically displayed in Figure 4 (Panel A for patients with paroxysmal AF and Panel B patients with persistent AF). For patients with paroxysmal AF, the 30-day monitoring strategy had the highest estimates for sensitivity (64.8\%) and NPV (50.4\%), whereas for patients with persistent AF, the quarterly 7-day monitoring strategy had the highest estimates for sensitivity (71.8\%) and NPV (54.1\%\%) for detecting AF events $>6$ min. A single 7-day monitor (a strategy commonly used in clinical practice) had a low sensitivity (45.2\% and $43.4 \%)$ and negative predictive value $(39.5 \%$ and $37 \%$ ) for detecting $\mathrm{AF}$ events $>6$ min in patients with paroxysmal and persistent AF, respectively. Heat maps showing daily AF burden within 1 year post blanking period in patients with paroxysmal and persistent AF are shown in Supplement Figure 1 and Supplement Figure 2, respectively.

\section{Discussion}

This study represents the largest, "real-world" analysis of AF ablation outcomes in patients with continuous, pre- and post-ablation monitoring using a CIED showing how the measure and definition of recurrence following ablation can alter interpretation of procedural success. In short, the incidence of any perceived recurrence of AF following ablation varies substantially depending on definitions of the minimum duration of required $\mathrm{AF}$ used to define procedural failure and the surveillance method by which the outcome is assessed. Success rates quoted in the published literature vary substantially depending on the above factors as well as whether patients had paroxysmal or persistent AF at baseline, the ablation technique, the number of ablations and presence of antiarrhythmic drugs, with recurrences in the first year after the blanking period being most commonly reported between 60 to $75 \%^{20,21,22}$.

Detection of AF recurrence following ablation is significantly influenced by the method of monitoring, as is shown in the monitoring strategy simulation. The strategies of a single 24-hour, 48-hour or 7-day monitor had the poorest test characteristics, especially for AF events $>6$ min where sensitivity and NPV were $<$ $50 \%$, worse than an unbiased coin flip. These results are likely due to patients having their first recurrences 
distributed over the year following the blanking period, and when these AF recurrences occur, they tend to cluster over consecutive days, as is shown in the heat maps (Supplement Figure 1 and 2). A 2018 metaanalysis of 66 studies (6941 patients) of AF ablation success in paroxysmal AF found that the method of $\mathrm{AF}$ detection following ablation was predictive of whether recurrence was detected rather than the specifics of the ablation procedure itself, with studies using long term CIEDs for monitoring being associated with higher perceived treatment failures than those using intermittent discrete monitoring methods ${ }^{13}$.

Our findings are concordant with the LINQ AF study, which enrolled 419 patients undergoing radiofrequency (RF) ablation to have a LINQ implantable loop recorder implanted following the procedure. They found success rate varied with the definition of AF recurrence from $46-79 \%$, findings overall similar to our study, and the authors suggest that $>6$ minutes of AF after a 90 days blanking and/or an AF burden $>0.1 \%$ be used in future studies as measure of clinically significant success ${ }^{10}$.

While historically most clinical trials of $\mathrm{AF}$ ablation have used time to first recurrence as an endpoint $^{3,4,23,24,25}$ due to relative ease of measurement, AF burden may be a more objective and clinically relevant endpoint ${ }^{2,12,26,11}$. In the CIRCA-DOSE trial, 346 paroxysmal AF patients were randomized 1:1:1 to RF pulmonary vein isolation (PVI), 2minute cryoballoon PVI or 4 minute cryoballoon PVI at least 30 days after implantable loop recorder implantation. At 12 months, despite only $51.7 \%-53.9 \%$ of patients being free of AF, AF burden was reduced by $98.4-99.9 \%{ }^{14}$. These results are similar to what was found in the present study, where in patients with paroxysmal AF, the survival estimates for the event $\mathrm{AF}>6$ minutes at one year was $28.2 \%$ but had a reduction of $99.6 \%$ in the overall median percentage of time in AF. Similarly, in patients with persistent AF, despite the survival estimate at one year of only $24.9 \%$ for AF events $>6$ minutes, there was a $99.3 \%$ reduction in the overall median percentage of time in AF.

While improvement in symptoms is the major clinical endpoint of ablation for AF, AF duration and burden appear to correlate with risk of stroke ${ }^{15,16,18,27}$, raising the hypothesis that strategies that reduce AF burden could potentially mitigate stroke risk, even in the absence of completely eliminating recurrent AF. This hypothesis, along with the data that AF burden reduction may similarly reduce heart failure hospitalization and mortality was tested in a recent random-effect model meta-analysis of large databases, registries and a single randomized control trial (the CABANA trial ${ }^{1}$ ) with a total of 241,372 patients. It showed that catheter ablation was associated with a decreased risk of mortality (hazard ratio [HR] 0.62), stroke (HR 0.63 ) and hospitalization for heart failure (HR 0.64) when compared to medical therapy alone ${ }^{19}$. While the CABANA trial individually was negative for the primary outcome of death, disabling stroke, serious bleeding, or cardiac arrest, it was subject to substantial crossover and low event rates, and the per-protocol analysis findings are largely consistent with the findings of the aforementioned meta-analysis ${ }^{1}$.

This analysis has a number of important limitations. It is retrospective and observational in nature; thus, unmeasured confounding cannot be eliminated. The population studied were by definition required to have had prior Medtronic CIEDs implanted, thus introducing significant selection bias in this group, therefore the results may not be generalizable to patients without implanted CIEDs. Furthermore, we do not have accurate data on antiarrhythmic drug use post-ablation, so the actual success rates of ablation alone may be overestimated. Lastly, in order to reduce the false positive rate associated with short unadjudicated AF episodes found on CIEDs, the minimum duration of AF evaluated was 6 minutes, longer than the 30 second threshold recommended in guidelines.

\section{Conclusion}

In this real-world de-identified EHR dataset of AF patients with CIEDs undergoing catheter ablation, perceived treatment success varied substantially with different definitions of minimally required AF duration and the method of recurrence detection. The traditional metrics of absolute recurrence or time to first $\mathrm{AF}$ recurrence following ablation undervalues the efficacy of ablation when compared to the measure of $\mathrm{AF}$ burden reduction, which is likely a more clinically relevant endpoint.

\section{Acknowledgements}


We would like to acknowledge Medtronic and Optum_( for use of their data.

\section{Authorship Contributions}

All Authors contributed equally to the preparation of the manuscript.

Disclosure of Conflicts of Interest

Rod Passman is a consultant to Medtronic and Abbott, receives research support from Pfizer and receives royalties from UpToDate. Paul Ziegler and João Monteiro are employees and shareholders of Medtronic. Graham Lohrmann and Rachel Kaplan have no disclosures.

\section{References}

1. Packer DL, Mark DB, Robb RA et al. Effect of Catheter Ablation vs Antiarrhythmic Drug Therapy on Mortality, Stroke, Bleeding, and Cardiac Arrest among Patients with Atrial Fibrillation: The CABANA Randomized Clinical Trial. In: JAMA - Journal of the American Medical Association. Vol 321. American Medical Association; 2019:1261-1274. doi:10.1001/jama.2019.0693

2. Steven D, Rostock T, Lutomsky B et al. What is the real atrial fibrillation burden after catheter ablation of atrial fibrillation? A prospective rhythm analysis in pacemaker patients with continuous atrial monitoring. Eur Heart J . 2008;29(8):1037-1042. doi:10.1093/eurheartj/ehn024

3. Packer DL, Kowal RC, Wheelan KR et al. Cryoballoon ablation of pulmonary veins for paroxysmal atrial fibrillation: First results of the North American arctic front (STOP AF) pivotal trial. J Am Coll Cardiol . 2013;61(16):1713-1723. doi:10.1016/j.jacc.2012.11.064

4. Nielsen JC, Johannessen A, Raatikainen P et al. Long-term efficacy of catheter ablation as first-line therapy for paroxysmal atrial fibrillation: 5-year outcome in a randomised clinical trial.Heart . 2017;103(5):368-376. doi:10.1136/heartjnl-2016-309781

5. Mark DB, Anstrom KJ, Sheng S et al. Effect of Catheter Ablation vs Medical Therapy on Quality of Life among Patients with Atrial Fibrillation: The CABANA Randomized Clinical Trial. In: JAMA Journal of the American Medical Association. Vol 321. American Medical Association; 2019:1275-1285. doi:10.1001/jama.2019.0692

6. Blomström-Lundqvist C, Gizurarson S, Schwieler J et al. Effect of Catheter Ablation vs Antiarrhythmic Medication on Quality of Life in Patients with Atrial Fibrillation: The CAPTAF Randomized Clinical Trial.JAMA - J Am Med Assoc . 2019;321(11):1059-1068. doi:10.1001/jama.2019.0335

7. Marrouche NF, Brachmann J, Andresen D et al. Catheter ablation for atrial fibrillation with heart failure. $N$ Engl J Med . 2018;378(5):417-427. doi:10.1056/NEJMoa1707855

8. Di Biase L, Mohanty P, Mohanty S et al. Ablation Versus Amiodarone for Treatment of Persistent Atrial Fibrillation in Patients With Congestive Heart Failure and an Implanted Device: Results From the AATAC Multicenter Randomized Trial. In: Circulation. Vol 133. Lippincott Williams and Wilkins; 2016:1637-1644. doi:10.1161/CIRCULATIONAHA.115.019406

9. Calkins H, Kuck KH, Cappato R et al. 2012 HRS/EHRA/ECAS Expert Consensus Statement on Catheter and Surgical Ablation of Atrial Fibrillation: Recommendations for Patient Selection, Procedural Techniques, Patient Management and Follow-up, Definitions, Endpoints, and Research Trial Design. Heart Rhythm . 2012;9(4):632-696.e21. doi:10.1016/j.hrthm.2011.12.016

10. Wechselberger S, Kronborg M, Huo Y et al. Continuous monitoring after atrial fibrillation ablation: the LINQ AF study. EP Eur . 2018;20(FI_3):f312-f320. doi:10.1093/europace/euy038

11. Dinshaw L, Schäffer B, Akbulak Ö et al. Long-term efficacy and safety of radiofrequency catheter ablation of atrial fibrillation in patients with cardiac implantable electronic devices and transvenous leads. $J$ Cardiovasc Electrophysiol . 2019;30(5):679-687. doi:10.1111/jce.13890 
12. Heeger $\mathrm{CH}$, Tscholl V, Salloum $\mathrm{O}$ et al. What is the real recurrence rate after cryoballoon-based pulmonary vein isolation? Lessons from rhythm follow-up based on implanted cardiac devices with continuous atrial monitoring. Hear Rhythm . 2018;15(12):1844-1850. doi:10.1016/j.hrthm.2018.07.016

13. Ferreira-Martins J, Howard J, Al-Khayatt B et al. Outcomes of paroxysmal atrial fibrillation ablation studies are affected more by study design and patient mix than ablation technique. J Cardiovasc Electrophysiol . 2018;29(11):1471-1479. doi:10.1111/jce.13745

14. Andrade JG, Champagne J, Dubuc M et al. Cryoballoon or Radiofrequency Ablation for Atrial Fibrillation Assessed by Continuous Monitoring: A Randomized Clinical Trial. Circulation . 2019;140(22):17791788. doi:10.1161/CIRCULATIONAHA.119.042622

15. Kaplan RM, Koehler J, Ziegler PD, Sarkar S, Zweibel S, Passman RS. Stroke risk as a function of atrial fibrillation duration and CHA2DS2-VASc score. Circulation . 2019;140(20):1639-1646. doi:10.1161/CIRCULATIONAHA.119.041303

16. Healey JS, Connolly SJ, Gold MR et al. Subclinical Atrial Fibrillation and the Risk of Stroke. $N$ Engl J Med . 2012;366(2):120-129. doi:10.1056/NEJMoa1105575

17. Glotzer T V., Daoud EG, Wyse DG et al. The Relationship between daily atrial tachyarrhythmia burden from implantable device diagnostics and stroke risk the trends study. Circ Arrhythmia Electrophysiol . 2009;2(5):474-480. doi:10.1161/CIRCEP.109.849638

18. Steinhubl SR, Waalen J, Edwards AM et al. Effect of a home-Based wearable continuous ECG monitoring patch on detection of undiagnosed atrial fibrillation the mSToPS randomized clinical trial. JAMA - J Am Med Assoc . 2018;320(2):146-155. doi:10.1001/jama.2018.8102

19. Saglietto A, De Ponti R, Di Biase L et al. Impact of atrial fibrillation catheter ablation on mortality, stroke, and heart failure hospitalizations: A meta-analysis. J Cardiovasc Electrophysiol . Published online March 1, 2020. doi:10.1111/jce.14429

20. Jais P, Cauchemez B, Macle L et al. Catheter ablation versus antiarrhythmic drugs for atrial fibrillation: The A4 study. Circulation . 2008;118(24):2498-2505. doi:10.1161/CIRCULATIONAHA.108.772582

21. Calkins H, Reynolds MR, Spector P et al. Treatment of atrial fibrillation with antiarrhythmic drugs or radiofrequency ablation: Two systematic literature reviews and meta-analyses. Circ Arrhythmia Electrophysiol . 2009;2(4):349-361. doi:10.1161/CIRCEP.108.824789

22. Calkins H, Hindricks G, Cappato R et al. 2017 HRS/EHRA/ECAS/APHRS/SOLAECE expert consensus statement on catheter and surgical ablation of atrial fibrillation. Hear Rhythm . 2017;14(10):e275-e444. doi:10.1016/j.hrthm.2017.05.012

23. Boersma LVA, Castella M, Van Boven W et al. Atrial fibrillation catheter ablation versus surgical ablation treatment (FAST): A 2-center randomized clinical trial. Circulation . 2012;125(1):23-30. doi:10.1161/CIRCULATIONAHA.111.074047

24. Di Biase L, Burkhardt JD, Mohanty P et al. Left Atrial Appendage Isolation in Patients With Longstanding Persistent AF Undergoing Catheter Ablation: BELIEF Trial. J Am Coll Cardiol . 2016;68(18):1929-1940. doi:10.1016/j.jacc.2016.07.770

25. Kuck K-H, Brugada J, Furnkranz A et al. Cryoballoon or Radiofrequency Ablation for Paroxysmal Atrial Fibrillation. N Engl J Med . 2016;374(23):2235-2245. doi:10.1056/NEJMoa1602014

26. Dulai R, Sulke N, Furniss S, Veasey RA. The effect of second-generation cryoablation without electrical mapping in persistent AF using continuous monitoring. J Interv Card Electrophysiol. Published online March 9, 2020. doi:10.1007/s10840-020-00721-1

27. Glotzer T V., Daoud EG, Wyse DG et al. The Relationship between daily atrial tachyarrhythmia burden from implantable device diagnostics and stroke risk the trends study. Circ Arrhythmia Electrophysiol 
. 2009;2(5):474-480. doi:10.1161/CIRCEP.109.849638

Tables:

Table 1. Baseline Characteristics.

\begin{tabular}{llll}
\hline Patient Characteristics & Paroxysmal AF $(\mathrm{n}=248)$ & Persistent AF $(\mathrm{n}=417)$ & Total $(\mathrm{n}=665)$ \\
\hline Age (years) & $66.2 \pm 9.3$ & $67.3 \pm 9.0$ & $66.6 \pm 8.9$ \\
Male & $181(73.0 \%)$ & $307(73.6 \%)$ & $488(73.4 \%)$ \\
Caucasian & $241(97.2 \%)$ & $394(94.5 \%)$ & $635(95.5 \%)$ \\
Hypertension & $208(83.9 \%)$ & $372(89.2 \%)$ & $580(87.2 \%)$ \\
Prior Stroke/TIA & $65(26.2 \%)$ & $95(22.8 \%)$ & $160(24.1 \%)$ \\
Heart Failure & $109(44.0 \%)$ & $247(59.2 \%)$ & $356(53.5 \%)$ \\
Diabetes & $72(29.0 \%)$ & $144(34.5 \%)$ & $216(32.5 \%)$ \\
Vascular Disease & $49(19.8 \%)$ & $102(24.5 \%)$ & $151(22.7 \%)$ \\
CHA $_{2}$ DS $_{2}$-VASc score & $3.3 \pm 1.9$ & $3.7 \pm 1.8$ & $3.5 \pm 1.8$ \\
Device type & & & \\
PPM & $73(29.4 \%)$ & $113(27.1 \%)$ & $186(28.0 \%)$ \\
ICD & $38(15.3 \%)$ & $104(24.9 \%)$ & $142(21.4 \%)$ \\
CRT-P & $2(0.8 \%)$ & $6(1.4 \%)$ & $8(1.2 \%)$ \\
CRT-D & $13(5.2 \%)$ & $75(18.0 \%)$ & $88(13.2 \%)$ \\
ICM & $122(49.2 \%)$ & $119(28.5 \%)$ & $241(36.2 \%)$ \\
\hline
\end{tabular}

Continuous variables displayed as mean \pm standard deviation, categorical variables displayed as number (\%), persistent $\mathrm{AF}$ patients were required to have 7 consecutive days with $>23$ hours AF burden.

AF: Atrial fibrillation; TIA: Transient ischemic attack; PPM: Permanent pacemaker; ICD: Implantable cardiac defibrillator; CRT-P: Cardiac resynchronization therapy pacemaker; CRT-D: Cardiac resynchronization therapy defibrillator; ICM: Implantable cardiac monitor.

\section{Figures:}

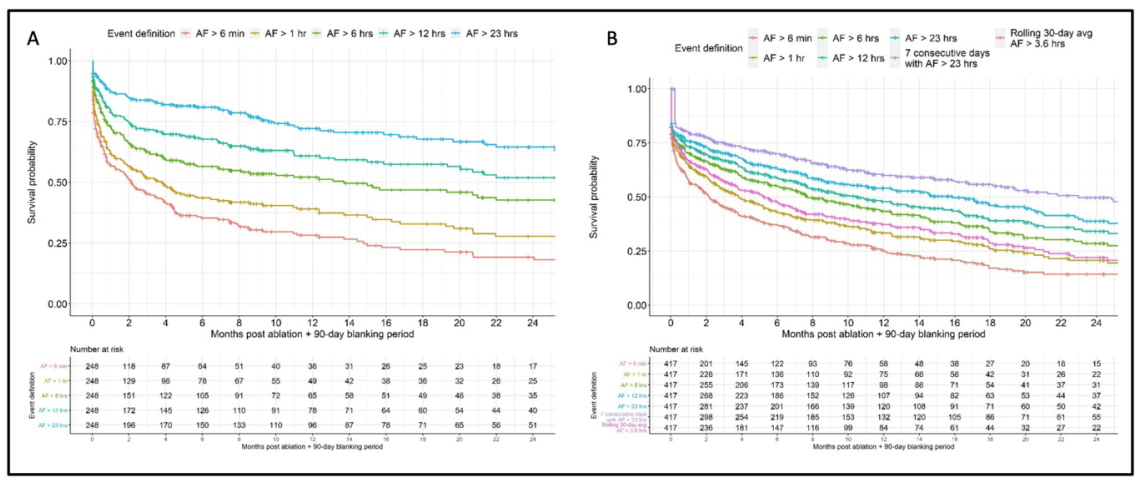

Figure 1. Freedom from Atrial Fibrillation (AF) Following Catheter Ablation Stratified by Event Definition in Patients with Paroxysmal AF (Panel A, $\mathrm{n}=248$ ) and Persistent AF (Panel B, $\mathrm{n}=417$ ). 


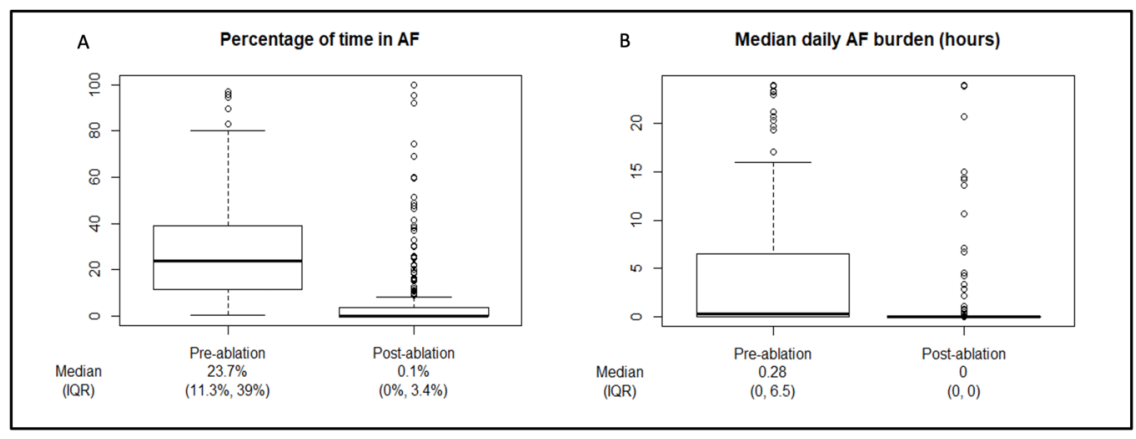

Figure 2. Reduction in Total AF Burden (Panel A) and Median Daily AF Burden (Panel B) in Patients with Paroxysmal AF Following Ablation.

AF: Atrial fibrillation; IQR: Interquartile range

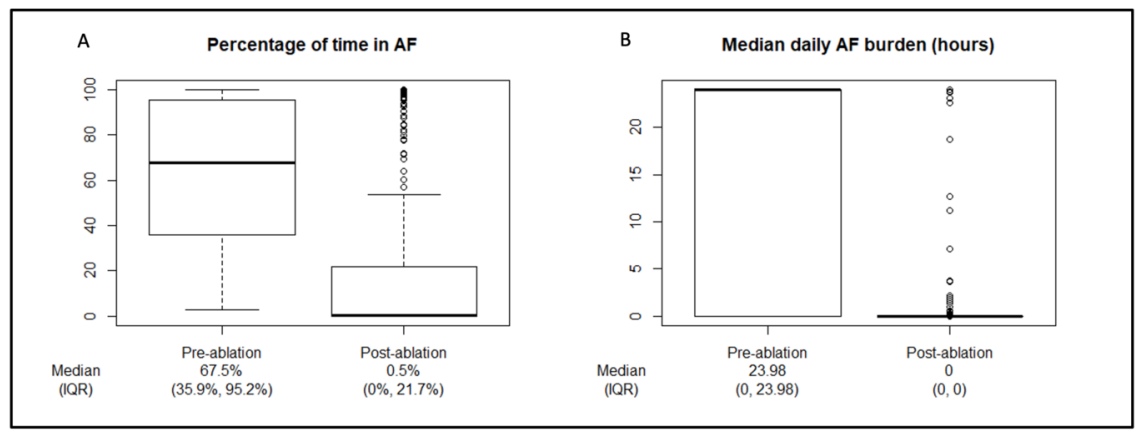

Figure 3. Reduction in Total AF Burden (Panel A) and Median Daily AF Burden (Panel B) in Patients with Persistent AF Following Ablation.

AF: Atrial fibrillation; IQR: Interquartile range

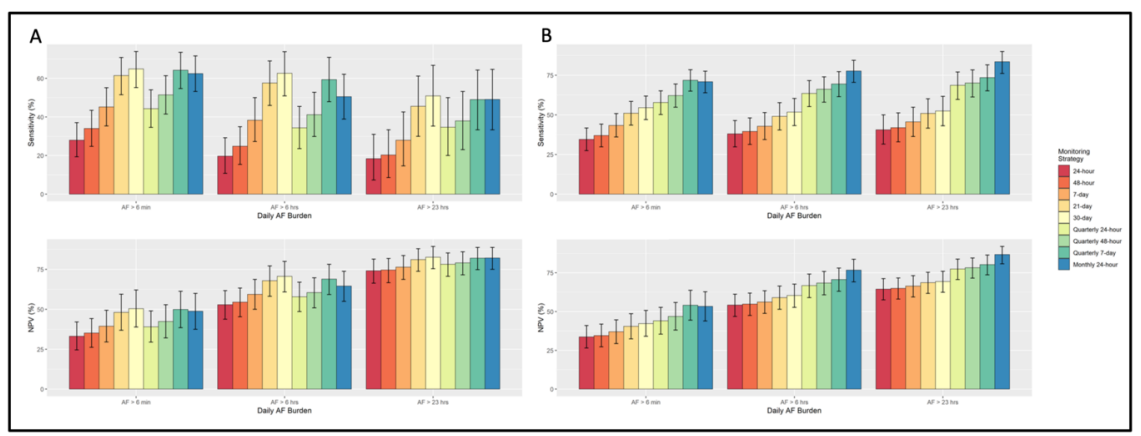

Figure 4. Estimated Sensitivity and NPV by Monitoring Strategy at 1 Year Post Blanking Period in Paroxysmal (Panel A, $\mathrm{n}=137$ ) and Persistent AF (Panel B, $\mathrm{n}=232$ ) Patients.

AF: Atrial fibrillation; NPV: Negative predictive value

\section{Supplement tables:}


Supplement Table 1: Diagnosis Codes Used to Detect Medical History 


\begin{tabular}{ll}
\hline Medical history & Diagnosis Codes (ICD-9/ICD-10) \\
\hline Hypertension & $401 . \mathrm{x}, 402 . \mathrm{x}, 403 . \mathrm{x}, 404 . \mathrm{x}, 405 . \mathrm{x}, \mathrm{I10.x}, \mathrm{I} 11 . \mathrm{x}$, \\
& $\mathrm{I} 12 . \mathrm{x}, \mathrm{I} 13 . \mathrm{x}, \mathrm{I} 15 . \mathrm{x}$ \\
Stroke/TIA & $433 . \mathrm{x}, 434 . \mathrm{x}, 435 . \mathrm{x}, 436 . \mathrm{x}, \mathrm{G} 45.0, \mathrm{G} 45.1, \mathrm{G} 45.2$, \\
& $\mathrm{G} 45.8, \mathrm{G} 45.9, \mathrm{Z} 86.73, \mathrm{~V} 12.54, \mathrm{I} 63 . \mathrm{x}, \mathrm{I} 65 . \mathrm{x}, \mathrm{I} 66 . \mathrm{x}$ \\
Heart Failure & $428 . \mathrm{x}, 402.01,402.11,402.91,404.01,404.03$, \\
& $404.11,404.13,404.91,404.93, \mathrm{I} 50 . \mathrm{x}, \mathrm{I} 11.0, \mathrm{I} 13.0$, \\
& $\mathrm{I} 13.2$ \\
Diabetes & $250 . \mathrm{x}, \mathrm{E} 10 . \mathrm{x}, \mathrm{E} 11 . \mathrm{x}$ \\
Vascular Diseases & $440.0,440.2 \mathrm{x}, 443.9, \mathrm{I} 70.0, \mathrm{I} 70.2 \mathrm{x}, \mathrm{I} 73.9$ \\
\hline
\end{tabular}

\section{Supplement figures:}

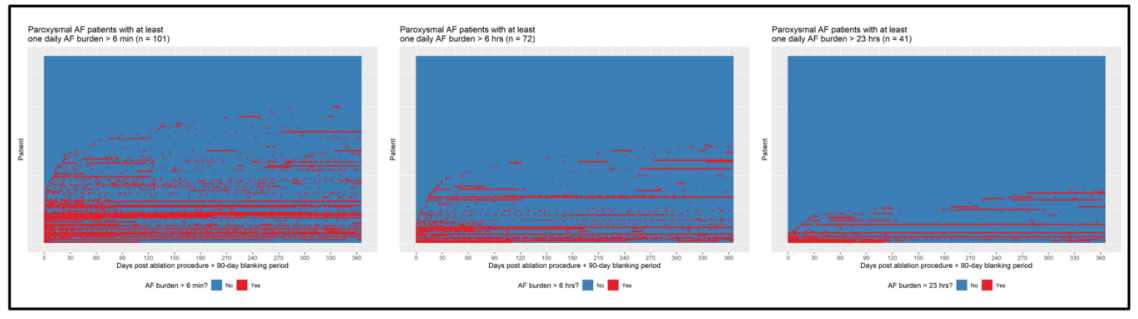

Supplement Figure 1. Heat Maps of Atrial Fibrillation Burden in Patients with Paroxysmal Atrial Fibrillation.

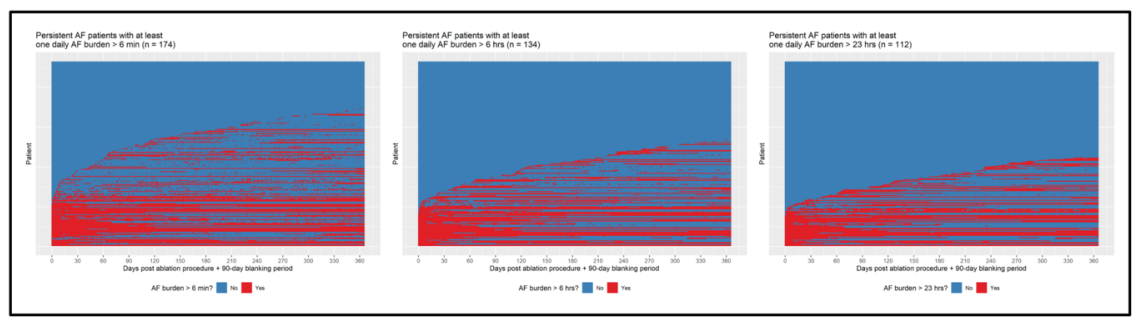

Supplement Figure 2. Heat Maps of Atrial Fibrillation Burden in Patients with Persistent Atrial Fibrillation. 